\title{
Análise Computadorizada da Cardiotocografia Anteparto em Gestações de Alto Risco
}

\author{
Computerized Antepartum Cardiotocography Analysis \\ in High Risk Pregnancies
}

Roseli Mieko Yamamoto Nomura, Rossana Pulcineli Vieira Francisco, Débora Simões Steinman, Seizo Miyadahira, Marcelo Zugaib

\begin{abstract}
RESUM0
Objetivos: analisar a freqüencia dos resultados das cardiotocografias computadorizadas realizadas em gestantes de alto risco e relacionar o critério proposto pelo sistema com os resultados perinatais.

Métodos: estudamos prospectivamente 233 gestantes de alto risco que realizaram 485 cardiotocografias computadorizadas. Foram excluidos casos de anomalias fetais e os exames com perda de sinal superior a 20\% (proporção de episódios de 3,75 milissegundos do traçado onde não se constata intervalo de pulso por perda de captação dos sinais de batimentos cardiacos fetais). Para estudo da associação da cardiotocografia com os resultados perinatais, analisou-se o último exame realizado na semana anterior ao parto (71 casos), excluindo-se casos com diagnóstico de diástole zero ou reversa na dopplervelocimetria das artérias umbilicais.

Resultados: após a exclusão de 33 exames com perda de sinal superior a 20\%, constatou-se que 404 cardiotocografias foram caracterizadas como normais (83,3\%). Quanto à duração do exame, em 62,1\% foi de até 20 minutos e em 79,0\% de até 30 minutos. A análise das correlações com os resultados perinatais demonstrou associação significativa $(p<0,05)$ entre a cardiotocografia computadorizada anormal e menor idade gestacional no nascimento, menor peso do RN, Apgar do $1^{\circ}$ minuto inferior a 7 (33\% dos casos) internação em UTI neonatal (55,5\%) e intubação do RN na sala de parto (44,4\%).

Conclusões: a cardiotocografia computadorizada com duração de até 30 minutos em gestações de alto risco revelou-se normal na maioria dos exames. Os casos que não preencheram os critérios de normalidade relacionaram-se significativamente com resultados perinatais adversos.
\end{abstract}

PALAVRAS-CHAVE: Cardiotocografia. Freqüência cardiaca fetal. Vitalidade fetal.

\section{Introdução}

Durante as três últimas décadas, a utilização de tecnologia avançada na Obstetrícia moderna permitiu o conhecimento detalhado da fisiologia fetal, de tal modo que o feto tornou-se um se-

Setor de Vitalidade Fetal

Clínica Obstétrica - Hospital das Clínicas

Faculdade de Medicina da Universidade de São Paulo

Correspondência:

Roseli Mieko Yamamoto Nomura

Rua General Canavarro, 280 - Bairro Campestre

09070-440 - Santo André - SP

Tel: (11)4991-2481 / 4221-8778; Fax: (11) 4221-8752

e-mail: roselinomura@uol.com.br

Home Page: http://www.hcnet.usp.br/ob/ gundo paciente, solicitando propedêutica especializada. Neste contexto, a cardiotocografia tem sido um dos métodos mais empregados na avaliação da vitalidade fetal ${ }^{1}$. Este exame se baseia na análise dos padrões da freqüência cardiaca fetal $(\mathrm{FCF})$, sendo efetuado pelo cardiotocógrafo, dispositivo que registra em traçados próprios a FCF e as contrações uterinas. Alterações nas características da FCF são observadas nos diversos estados comportamentais do feto (ciclo sono-vigília), nos distúrbios de sua oxigenação ou em situações decorrentes da utilização de medicações durante a gestação ${ }^{2}$. Pela facilidade, praticidade e inocuidade, próprias do exame cardiotocográfico, este método difundiu-se rapidamente no mundo 
e, atualmente, está presente nos centros que acompanham as gestações de alto risco.

A interpretação dos traçados cardiotocográficos pode ser realizada a partir de duas técnicas de análise: a visual e a computadorizada. A análise visual, aplicada desde a introdução do método, está sujeita a grande variabilidade de critérios e limitada reprodutibilidade dos resultados ${ }^{3}$. Tradicionalmente, esta análise da cardiotocografia baseia-se na observação visual de acelerações da FCF e na variabilidade da freqüência basal. Entretanto, a elevada taxa de resultados falso-positivos ${ }^{4}$ da cardiotocografia convencional estimulou a procura de novas formas de estudo do traçado. Para suprimir os possiveis erros oriundos da subjetividade e visando diminuir as variações intra-observador e inter-observador da avaliação visual, foi desenvolvido um sistema computadorizado de análise da FCF por Dawes et al..$^{5}$, que estabeleceram critérios próprios a serem utilizados. Características detalhadas do traçado cardiotocográfico passaram a ser analisadas para determinação do prognóstico perinatal.

O desenvolvimento do sistema de análise computadorizada da FCF iniciou-se em $1978^{6}$, e os primeiros resultados encorajaram os autores ao aperfeiçoamento e aprimoramento desta tecnologia. Foi necessária, portanto, a padronização do sistema para a interpretação de novos parâmetros. O sistema computadorizado de análise do traçado cardiotocográfico deriva de critérios de normalidade da FCF baseados na análise de 48.000 exames ${ }^{7}$. Neste sistema, o sinal é captado pelo cardiotocógrafo que está conectado ao computador, onde um programa realiza a interpretação do traçado utilizando-se de software específico.

A análise computadorizada baseia-se no estudo da duração dos intervalos de tempo (em milissegundos) que decorre entre sucessivos batimentos cardiacos fetais. O sistema 8002 analisa o traçado cardiotocográfico em períodos de 3,75 segundos (1/16 de minuto), calculando a FCF média em cada período e também as diferenças entre períodos adjacentes. Um dos parâmetros introduzido pela análise computadorizada consiste na variação de curto prazo, que corresponde à média das diferenças dos valores da FCF média de períodos de 3,75 segundos adjacentes (analisado em intervalos de pulso). A redução da variação de curto prazo relaciona-se com a ocorrência de acidose metabólica ou óbito do feto ${ }^{8,9}$. Este tipo de análise avalia a variabilidade da FCF, principalmente a microoscilação, que não é mensurável na análise visual da cardiotocografia. A duração dos episódios de baixa variação da FCF, caracterizada quando, em 5 de 6 minutos consecutivos, a variação pico a pico da FCF por minuto, em intervalos de pulso, é inferior a 30 milisegundos, também pode ser utilizada na predição do valor do $\mathrm{pH}$ do sangue da artéria umbilical no nascimento ${ }^{10}$.

A literatura nacional apresenta poucos estudos sobre cardiotocografia computadorizada, principalmente pela dificuldade na interpretação dos critérios propostos pelo sistema. Este estudo tem como objetivos relatar a freqüência dos resultados observados neste exame, realizado em gestações de alto risco, descrevendo as principais características dos parâmetros avaliados, e analisar as relações entre o critério proposto pelo sistema e os resultados perinatais.

\section{Pacientes e Métodos}

Este trabalho foi desenvolvido de forma prospectiva no Setor de Vitalidade Fetal da Clínica Obstétrica do HC-FMUSP. A população estudada foi selecionada de acordo com os seguintes critérios de inclusão: gestantes de alto risco internadas na enfermaria da Clínica Obstétrica, com feto vivo e ausência de anomalias cromossômicas ou estruturais. Foram excluídos os exames nos quais houve perda de captação do sinal da FCF superior a 20\% (proporção de episódios de 3,75 milissegundos do traçado onde não se constata intervalo de pulso por perda de captação dos sinais de batimentos cardíacos fetais) ${ }^{7}$, e os casos nos quais o cálculo da idade gestacional era duvidoso. O limite de $20 \%$ foi adotado arbitrariamente para este estudo ${ }^{11}$.

No período compreendido entre janeiro de 2000 e agosto de 2001, 233 gestantes de alto risco foram avaliadas, sendo realizadas um total de 485 cardiotocografias computadorizadas. As pacientes apresentavam média de idade de 27,7 anos (desvio padrão de 8,8 anos) e 73 eram nulíparas $(31,3 \%)$. As gestantes incluídas neste estudo apresentavam as seguintes intercorrências: sindromes hipertensivas $(28,3 \%)$, diabete $(16,3 \%)$, colagenoses $(12,0 \%)$, cardiopatias $(9,0 \%)$, trabalho de parto prematuro $(6,7 \%)$, restrição do crescimento fetal $(6,4 \%)$, natimorto anterior $(3,0 \%)$, nefropatias $(3,0 \%)$, tiroidopatias $(3,0 \%)$, gemelaridade $(2,6 \%)$, redução do volume de líquido amniótico $(2,1 \%)$, aloimunização Rh $(1,7 \%)$, placenta prévia $(1,3 \%)$, amniorrexe prematura $(1,3 \%)$ e outros diagnósticos com incidência inferior a $1,0 \%$ em 18 casos.

Para descrever as principais características dos parâmetros avaliados pelo critério proposto pelo sistema computadorizado, foram incluídos todos os exames realizados. Para o estudo das relações entre o exame e os dados perinatais foram excluidos os casos que apresentavam intervalo superior a sete dias entre a data de realização da última 
cardiotocografia e o dia do parto, bem como as gestações que apresentavam diagnóstico de diástole zero ou reversa na dopplervelocimetria da artéria umbilical. Este subgrupo de pacientes era constituído de 71 gestantes, com média de idade de 28,3 anos (desvio padrão de 8,3 anos), das quais 22 eram nulíparas $(31,0 \%)$. As intercorrências maternas mais freqüentes foram: sindromes hipertensivas $(36,6 \%)$, diabete $(21,1 \%)$, cardiopatia $(12,7 \%)$, colagenoses $(8,5 \%)$ e redução do volume do líquido amniótico $(7,0 \%)$.

A cardiotocografia computadorizada foi realizada no período anteparto, utilizando-se monitor fetal da marca Sonicaid, com software para análise computadorizada da FCF (sistema 8002). Este software permite a análise dos seguintes parâmetros: variação de curto prazo, episódios de alta e baixa variação, FCF basal, acelerações e desacelerações da FCF, perda de sinal, contrações uterinas e movimentos fetais registrados pela gestante ${ }^{6}$.

O sistema é padronizado para ser utilizado somente no período anteparto. Nos primeiros 10 minutos de realização do exame, o sistema computadorizado não realiza nenhuma interpre- tação. Após este período, o programa realiza a primeira análise do traçado, que é revisto a cada dois minutos e apresentado na tela do vídeo, até que se atinja o critério de normalidade pré-estabelecido por Dawes et al. ${ }^{5}$. A cada análise, o sistema emite um sinal sonoro. O período máximo para realização do traçado é de 60 minutos. Ao término deste periodo, o programa finaliza-se automaticamente e inicia a impressão do laudo. Para idades gestacionais inferiores a 24 semanas e superiores a 42 semanas, os cálculos são realizados considerando-se como referência os padrões usados para as idades gestacionais de 24 e 42 semanas, respectivamente ${ }^{12}$.

O traçado cardiotocográfico é caracterizado como normal quando o critério de Dawes et al. é atingido. As condições normais que devem estar presentes para que este critério seja preenchido estão listadas e explicadas na Tabela 1 . No presente estudo, todos os exames foram interrompidos somente após o sistema sinalizar o preenchimento dos critérios, caso contrário, o exame prolongou-se até completar o tempo máximo de exame (60 minutos).

Tabela 1 - Condições de normalidade que devem estar presentes no traçado para que o critério de Dawes et al5. seja atingido na cardiotocografia computadorizada.

\begin{tabular}{ll}
\hline Condição & Normal \\
\hline Episódio de alta variação & No mínimo 1 episódio \\
Desacelerações & Nenhuma superior a 20 'lost beats' \\
FCF basal & Entre $116 \mathrm{bpm}$ e $160 \mathrm{bpm}$ em traçados com duração inferior a 30 minutos \\
Movimentos fetais ou acelerações da FCF & Um movimento fetal ou 3 acelerações da FCF \\
Padrão sinusoidal & Não devem existir evidências de ritmo sinusoidal \\
Variação de curto prazo & Deve ser $\geq 3$ milissegundos \\
Variabilidade em episódios de alta variação & Deve ocorrer uma aceleração ou a variabilidade deve ser superior ao percentil 10 \\
& com estimativa da contagem de movimentos fetais (por hora) superior a 20 \\
Erros & Não devem ocorrer erros ou desacelerações \\
& no final do traçado em análise
\end{tabular}

A variaçãzo de curto prazo ou STV (short term variation) da FCF corresponde à média das diferenças dos valores da FCF média (expressa por intervalo de pulso em milissegundos) observada entre períodos adjacentes de 3,75 segundos ${ }^{13}$. Define-se como episódio de alta variação uma seção do traçado onde pelo menos cinco de seis minutos consecutivos apresentam variação da FCF (em intervalo de pulso) pico a pico, por minuto, superior a 32 milissegundos. O limite para baixa variação é de 30 milissegundos, e períodos do traçado podem ficar sem definição quanto ao tipo da variação da $\mathrm{FCF}^{7}$. Para que se preencha o critério de análise da alta variação, é necessário que o traçado apresente pelo menos um episódio de alta variação, cujo valor da variabilidade (em $\mathrm{bpm}$ ) exceda o percentil 1 de acordo com a curva de distribuição normal para a idade gestacional ${ }^{7}$. Esta análise é realizada automaticamente pelo sistema computadorizado, comparando a variabilidade no período de alta variação com os valores do percentil 1 para cada idade gestacional. O sistema identifica as acelerações da FCF quando detecta aumento de 10 ou $15 \mathrm{bpm}$ em relação à linha de base $\mathrm{b}^{7}$. Para o traçado ser considerado normal, deve ocorrer uma aceleração ou a variabilidade da FCF nos 
episódios de alta variação deve ser maior que o percentil 10, de acordo com a curva de distribuição normal para a idade gestacional e o número de movimentos fetais superior a 20. Estas análises são realizadas pelo sistema e a interpretação dos critérios são impressos no laudo final do exame.

Foram estudados os seguintes resultados perinatais: tipo de parto, idade gestacional no nascimento, índices de Apgar do $1^{\circ}$ e $5^{\circ}$ minutos, peso do recém-nascido (RN), adequação do peso do RN à idade gestacional, intubação do RN na sala de parto e internação em unidade de terapia intensiva (UTI) neonatal. Os RN foram classificados como pequeno para a idade gestacional (PIG) se o peso fosse inferior ao percentil 10 para a idade gestacional, de acordo com a curva de normalidade desenvolvida pelo setor de neonatologia deste hospital ${ }^{14}$.

A idade gestacional foi calculada a partir da data da última menstruação (DUM), se esta era compativel com exame ultra-sonográfico realizado até a $20^{a}$ semana. Se a paciente não soubesse referir a DUM ou houvesse discordância com o exame ultra-sonográfico, a datação da gestação baseava-se na primeira ultra-sonografia realizada.

Foram analisadas as freqüências das características da cardiotocografia computadorizada de acordo com a análise efetuada pelo sistema. Para as variáveis qualitativas utilizou-se o teste de $\chi^{2}$ com correção de Yates para continuidade e, quando pertinente, o teste exato de Fisher. Para as variáveis quantitativas, utilizou-se o teste $t$ de Student. Adotou-se como nível de significância o valor $0,05(\alpha=5 \%)$. Com isso, niveis descritivos (p) inferiores a esse valor foram considerados significantes $(p<0,05)$.

Este estudo foi aprovado pela comissão de Ética para Análise de Projetos de Pesquisa (CAPPesq) do HC-FMUSP, e as pacientes concordaram em participar após esclarecimento dos objetivos da pesquisa.

\section{Resultados}

Um total de 485 cardiotocografias computadorizadas foram realizadas no período analisado. A média de exames realizados por paciente foi de 2,2, com desvio padrão de 3,0 exames. Em 404 exames $(83,3 \%)$, os critérios de Dawes et al. foram atingidos, caracterizando o exame como normal, e em $81(16,7 \%)$ os mesmos não foram atingidos. Após a exclusão de 33 exames $(6,8 \%)$ com perda de sinal superior a $20 \%$, observou-se que em 385 exames $(85,2 \%)$ os critérios foram preenchidos e em 67 não $(14,8 \%)$.

Quando analisamos o tempo de execução dos exames, constatamos, no grupo em que os critérios foram atingidos, média de tempo de duração do exame de 21,4 minutos, com desvio padrão de 13,5 . O tempo máximo necessário para que os critérios fossem preenchidos foi de 10 minutos em 125 exames (32,5\%), 20 minutos em 239 exames (62,1\%), 30 minutos em 304 exames $(79,0 \%)$ e 45 minutos em 351 exames $(91,2 \%)$.

As características dos parâmetros analisados pelo sistema computadorizado, de acordo com o critério de Dawes et al., podem ser observadas na Tabela 2. Não se observou diferença significativa na percentagem de perda do sinal de acordo com o resultado da cardiotocografia computadorizada, com média de $3,1 \%$ nos exames que atingem o critério e de $4,1 \%$ nos que não atingem. Os demais parâmetros analisados apresentaram diferenças de acordo com o resultado final da interpretação do programa de computador, observandose nos exames categorizados como normais maior número de movimentos fetais registrados, maior proporção de exames com presença de acelerações da FCF de 10 bpm e também de 15 bpm, e menor proporção de exames com desacelerações da FCF.

A análise dos episódios de alta e de baixa variação demonstrou que todos os exames que atingiram os critérios de normalidade apresentaram episódios de alta variação, e a proporção de episódios de baixa variação foram mais freqüentes no em grupo que o critério não foi preenchido.

Para a análise das relações entre os resultados da cardiotocografia computadorizada e os dados perinatais, avaliaram-se os casos em que o exame foi realizado no máximo sete dias antes da data do parto. Foram excluídas as gestações com diagnóstico de diástole zero ou reversa à dopplervelocimetria das artérias umbilicais. Entre as 71 gestações analisadas, a cardiotocografia preencheu os critérios de Dawes et al. em 61 (85,9\%) casos, e em $10(14,1 \%)$ o critério não foi satisfeito. Este grupo apresentou média da idade materna de 28,3 anos, com desvio padrão de 8,3 . Vinte e duas gestantes eram primigestas $(31,0 \%)$ e as intercorrências clínicas constatadas com maior freqüência foram as sindromes hipertensivas, em $36,6 \%$ (26 casos).

As correlações entre os dados perinatais e os resultados da cardiotocografia computadorizada estão expostas na Tabela 3. As médias da idade gestacional no nascimento e do peso do recémnascido foram significativamente menores nos casos que não atingiram o critério. Do mesmo modo, observamos proporção significativamente maior de recém-nascidos que necessitaram de intubação ao nascimento $(44,4 \%)$ e de internação em UTI neonatal $(55,5 \%)$ nos casos em que o re- 
sultado da cardiotocografia computadorizada não preencheu os critérios de normalidade, quando comparados aos casos com exame normal. De modo semelhante, o grupo com a cardiotocografia anormal apresentou maior proporção de RN com índice de Apgar de $1^{\circ}$ minuto inferior a sete (33\%) quando comparado ao grupo com exame normal, em que esta alteração do índice foi observada em 8,3\% dos RN. Na análise do índice de Apgar de $5^{\circ}$ minuto, não foram constatadas diferenças significativas na proporção de RN com este índice alterado (inferior a sete) de acordo com o resultado da cardiotocografia, apesar de proporção maior ter sido observada no grupo com cardiotocografia anormal.

Tabela 2 - Características dos parâmetros da cardiotocografia computadorizada de acordo com o seu resultado.

\begin{tabular}{|c|c|c|}
\hline \multirow[t]{2}{*}{ Parâmetro } & \multicolumn{2}{|c|}{ Cardiotocografia computadorizada } \\
\hline & $\begin{array}{l}\text { Critério atingido } \\
\qquad(\mathrm{n}=385)\end{array}$ & $\begin{array}{l}\text { Critério não atingido } \\
\qquad(n=67)\end{array}$ \\
\hline Perda do sinal (\%, média $\pm \mathrm{DP})$ & $3,1 \pm 4,5$ & $4,1 \pm 4,7$ \\
\hline Movimentos fetais ( $n^{\circ} /$ hora, média $\pm D P$ ) & $40,0 \pm 47,8$ & $23,2 \pm 25,5$ \\
\hline FCF basal (bpm, média $\pm \mathrm{DP})$ & $138,3 \pm 9,3$ & $143,2 \pm 19,5$ \\
\hline Presença de acelerações >10 bpm & $78(98,2 \%)$ & $45(67,2 \%)$ \\
\hline Presença de acelerações >15 bpm & $252(65,5 \%)$ & $26(38,8 \%)$ \\
\hline Presença de desacelerações & $19(4,9 \%)$ & $23(34,3 \%)$ \\
\hline Duração dos episódio de alta variação (min, média \pm DP) & $9,3 \pm 5,0$ & $0,6 \pm 2,2$ \\
\hline Presença de episódio de alta variação & $385(100 \%)$ & $5(7,5 \%)$ \\
\hline Duração dos episódio de baixa variação (min, média \pm DP) & $5,7 \pm 9,1$ & $33,1 \pm 15,5$ \\
\hline Presença de episódio de baixa variação & $153(39,7 \%)$ & $66(98,5 \%)$ \\
\hline Variação de curto prazo (ms, média \pm DP) & $8,5 \pm 2,8$ & $5,3 \pm 3,9$ \\
\hline Variação de curto prazo inferior a 3,0 ms & $0(0 \%)$ & $7(10,4 \%)$ \\
\hline
\end{tabular}

Tabela 3 - Resultados perinatais de acordo com o resultado da cardiotocografia computadorizada.

\begin{tabular}{lcc}
\hline Resultado perinatal & \multicolumn{2}{c}{ Cardiotocografia computadorizada } \\
& Critério atingido & Critério não atingido \\
\hline Idade gestacional no parto (sem, média \pm DP) & $36,9 \pm 3,1$ & $32,6 \pm 4,4^{*}$ \\
Peso do recém-nascido (gramas, média \pm DP) & $2635,9 \pm 770,8$ & $1762,0 \pm 991,4^{*}$ \\
Recém-nascido PIG (n/total, \%) & $8 / 61(13,1 \%)$ & $3 / 10(30 \%)$ \\
Cesárea (n/total, \%) & $48 / 60(80 \%)$ & $9 / 9(100 \%)$ \\
Apgar do $1^{\circ}$ minuto <7 (n/total, \%) & $5 / 60(8,3 \%)$ & $3 / 9(33 \%)^{*}$ \\
Apgar do $5^{\circ}$ minuto <7 (n/total, \%) & $1 / 60(1,7 \%)$ & $1 / 9(11,1 \%)$ \\
Internação do RN em UTI neonatal (n/total, \%) & $9 / 60(15 \%)$ & $5 / 9(55,5 \%)^{*}$ \\
Intubação do RN na sala de parto (n/total, \%) & $3 / 60(5 \%)$ & $4 / 9(44,4 \%)^{*}$ \\
\hline
\end{tabular}

${ }^{*} p<0,05$

\section{Discussão}

Entre as provas de vitalidade fetal utilizadas no acompanhamento de gestações de alto risco, destaca-se a cardiotocografia anteparto. Esta modalidade de exame foi introduzida no meio obstétrico antes do advento da ultra-sonografia, e, apesar de ser aplicada há mais de 30 anos, ainda subsistem dificuldades na interpretação de seus traça- dos. A sua análise envolve, freqüentemente, critérios subjetivos com considerável variação interobservadores e intra-observadores ${ }^{15}$, prejudicando a reprodutibilidade deste método. Apesar das eventuais dificuldades na interpretação e da limitação na confiabilidade do método, este exame da propedêutica fetal é considerado importante para a assistência prestada às gestações de alto risco.

Freqüentemente, em gestações de alto risco, ocorrem situações nos quais torna-se neces- 
sária a escolha do melhor momento para a resolução da gravidez. Evidentemente, nas gestações próximas ao termo, a tomada de decisões é facilitada pela presença da maturidade fetal. Porém, em gestações longe do termo, em que a imaturidade fetal é comum, as condutas resolutivas intempestivas podem comprometer o prognóstico neonatal. Assim, a acurácia diagnóstica torna-se elemento fundamental na avaliação do estado de oxigenação fetal. Neste sentido, a análise computadorizada do traçado cardiotocográfico pode facilitar a padronização do atendimento oferecido, com a ressalva da necessidade de profundos conhecimentos a respeito deste método diagnóstico.

Tecnicamente, a cardiotocografia computadorizada demonstrou ser de fácil aplicação, sendo que a impressão de laudos em papel comum constitui aspecto interessante, reduzindo-se os custos na utilização do método. A possibilidade da utilização do sistema em rede oferece possibilidades de ampla acessibilidade aos dados, facilitando a visualização dos traçados a partir de qualquer terminal de computador interligado à rede local. Entretanto, o sistema foi elaborado para utilização somente no período anteparto, o que realmente limita a sua aplicação durante o trabalho de parto.

Quanto à duração do exame, a maioria dos casos finaliza em tempo adequado (até 20 minutos), não necessitando obrigatoriamente de profissional médico para a sua realização. Entretanto, para correta interpretação de seus resultados há necessidade de submetê-los à correlação clínica aplicada com conhecimentos da fisiopatologia fetal pertinente ${ }^{16}$, somente possivel pelo médico especializado. A perda do sinal acima do limite considerado adequado para a presente pesquisa ocorreu em pequena percentagem de casos $(6,8 \%)$. Sabe-se que a perda do sinal prejudica a análise do traçado pelo sistema computadorizado, e perdas superiores a $25 \%$ resultam em análise duvidosa do traçado 7 .

A realização da cardiotocografia computadorizada torna-se interessante para fetos em idades gestacionais precoces, em que a imaturidade do sistema nervoso autônomo pode influenciar o padrão cardiotocográfico com menor incidência de acelerações da FCF. O sistema computadorizado permite análise da variação da FCF, utilizando critérios que dispensam a presença de acelerações transitórias para efetuar o diagnóstico de normalidade, valendo-se principalmente da ocorrência de episódios de alta variação ${ }^{8}$. A ocorrência destes episódios de alta variação da FCF demonstrou apresentar maior sensibilidade para o diagnóstico de normalidade do teste ${ }^{6}$. Nossos resultados demonstram que entre os exames caracterizados como normais, ao preencherem os critérios pro- postos, 54,5\% não apresentavam acelerações transitórias (de $15 \mathrm{bpm}$ ) e 1,7\% não apresentavam acelerações de $10 \mathrm{bpm}$ (Tabela 2). Nos traçados que não se caracterizaram como normais pelo sistema computadorizado, 38,8\% apresentavam acelerações transitórias, o que contrasta com os critérios adotados classicamente na avaliação visual, na qual a presença de acelerações transitórias classifica o traçado como normal ${ }^{1}$. As gestações de alto risco que apresentem intercorrências longe do termo beneficiam-se com a aplicação deste método de avaliação da cardiotocografia, pois, em períodos em que sabidamente a incidência de acelerações da FCF é menor, a análise baseada na variação da FCF poderá fornecer melhor subsídio clínico para o seguimento da gestação ${ }^{17}$.

Os resultados obtidos neste estudo demonstram maior freqüência de movimentos fetais e menor valor de FCF basal quando o critério de Dawes et al. é atingido. Considerando-se que o feto bem oxigenado apresenta movimentação corpórea adequada, a freqüência da ocorrência deste evento associa-se portanto à normalidade do exame. Também sabemos que a hipoxemia fetal pode provocar aumento da FCF, pela mediação do sistema nervoso simpático ${ }^{2}$. A observação de maior média na FCF no grupo cuja cardiotocografia computadorizada não preencheu os critérios propostos pode estar relacionada à maior incidência de fetos hipoxêmicos neste grupo.

A possibilidade da determinação da variação de curto prazo pelo sistema computadorizado oferece a avaliação de um parâmetro que não é passivel de mensuração na análise visual ${ }^{18}$. Este estudo da variabilidade instantânea auxilia no diagnóstico de casos com comprometimento extremo da oxigenação fetal, correlacionando-se com a ocorrência de acidose metabólica fetal ou óbito fetal ${ }^{8,9}$. Porém, esta anormalidade constitui alteração tardia da vitalidade. Street et $a .^{8}$ relatam que a análise da variação de curto prazo da FCF permite a detecção de fetos com acidemia metabólica ou casos que evoluam com óbito fetal. Dawes et al. ${ }^{9}$ relatam $34 \%$ de acidemia no nascimento, caracterizada pelo valor do excesso de bases superior a $12 \mathrm{mmol} / 1$, em casos com a variação de curto prazo inferior a 2,6 ms.

Os episódios de baixa variação da FCF não são contemplados dentro dos critérios propostos para caracterizar um exame como normal; entretanto, observa-se no presente estudo que estes episódios tornam-se muito freqüentes e com longa duração em exames que não preenchem os critérios de normalidade. Guzman et al. ${ }^{10}$ utilizam a duração destes episódios na predição do $\mathrm{pH}$ fetal no nascimento, demonstrando que a análise deste parâmetro merece atenção. 
A ocorrência de desacelerações tardias não é perfeitamente detectada pelo sistema, uma vez que somente desacelerações de maior área serão demarcadas no laudo final ${ }^{9,19,20}$. Contudo, fetos com comprometimento importante apresentando desacelerações tardias freqüentemente apresentam alterações na variabilidade da $\mathrm{FCF}$, que são avaliadas pelo sistema computadorizado a partir da detecção de episódios de baixa variação da FCF e da variação de curto prazo. Dawes et al. ${ }^{19}$ relatam que os casos em que o traçado cardiotocográfico apresenta desacelerações de maior área (superior a 20 'lost beats') apresentam $40 \%$ de chance de evoluir com variação de curto prazo de valores descendentes. Entretanto, a ocorrência de desacelerações na FCF sempre chama a atenção na interpretação do traçado, e a inadequada identificação destas pelo sistema reforça a idéia de que um profissional médico especializado acompanhe sempre a análise dos dados obtidos.

Neste estudo observamos associação significativa entre a ocorrência de cardiotocografia computadorizada anormal e resultados perinatais indesejáveis, principalmente no que diz respeito ao Apgar do primeiro minuto. Estes achados também são descritos na literatura, pelo que Dawes et al. ${ }^{20}$ concluem que o melhor indicador da condição fetal é a variação da $\mathrm{FCF}$, e que valores inferiores a 20 milissegundos na variação de longo prazo associam-se a acidemia fetal ou mortalidade perinatal. Anceschi et al. ${ }^{21}$ analisam gestações sem complicações, e observam que a presença de acelerações da FCF de $15 \mathrm{bpm}$ e a freqüência de movimentos fetais maior que 21 movimentos por hora na cardiotocografia computadorizada associam-se a bom resultado neonatal. Maior casuística, com estudo da gasometria do cordão umbilical no nascimento, permitirá avaliar o valor preditivo dos parâmetros desta modalidade da cardiotocografia para o diagnóstico de hipoxia fetal ${ }^{22}$.

A cardiotocografia computadorizada realizada em gestações de alto risco revelou-se normal na maioria das análises efetuadas, e a duração dos exames foi, com freqüência, inferior a $30 \mathrm{mi}-$ nutos e mesmo na ausência de acelerações transitórias, outras características do traçado permitem que fetos normais sejam identificados. As alterações do traçado detectadas pelo sistema associaram-se significativamente aos resultados perinatais alterados, e quando os critérios não são atingidos, caracteriza-se situação de risco para o comprometimento da vitalidade fetal, sendo indicada complementação da propedêutica fetal ${ }^{23}$. Nesta casuística, maiores taxas de morbidade neonatal imediata foram associadas aos resultados alterados. Senat et al. ${ }^{24}$ observaram correlação negativa entre os valores do índice de pulsatilidade da dopplervelocimetria do ducto venoso e a variação de curto prazo na cardiotocografia computadorizada. Os autores relatam que o índice de pulsatilidade do ducto venoso apresenta elevações de seus valores na hipoxia e acidemia fetais, e associa-se à redução significativa da variação de curto prazo nestas situações.

A utilização da cardiotocografia computadorizada no diagnóstico da condição fetal necessita ainda estudos que estabeleçam o papel deste exame propedêutico, com enfoques distintos para os diversos parâmetros analisados. Os casos que não preencheram os critérios de normalidade relacionaram-se significativamente com resultados perinatais adversos.

\section{ABSTRACT}

Purpose: to study computerized cardiotocography performed in high-risk pregnancies, analyze the results, and correlate the criteria to perinatal results.

Patients and Methods: two hundred and thirty-three highrisk pregnancies were studied prospectively, performing a total of 485 computerized cardiotocographies. The exclusion criteria included fetal anomalies and signal loss over 20\% (proportion of 3.75-millisecond periods in which there were no valid pulse intervals). The perinatal results of 71 pregnancies were correlated to the last cardiotocography, performed at least seven days before birth, excluding patients with absent or reversed end diastolic velocities in the umbilical arteries.

Results: thirty-three examinations with signal loss over $20 \%$ were excluded. The normal criteria were met in 404 (83.3\%), and $62.1 \%$ examinations met the criteria within 20 minutes and $79 \%$ within 30 minutes. The abnormal computerized cardiotocography was related significantly $(p<0.05)$ to adverse perinatal results, such as: preterm delivery, first minute Apgar score less than 7 (33\%), neonatal intensive care admission (55.5\%) and intubation of newborn at delivery $(44.4 \%)$.

Conclusions: computerized cardiotocography in high-risk pregnancies met the normal criteria in most of the cases, with the examination performed for 30 minutes. The cases that did not meet the criteria correlated significantly to adverse perinatal results.

KEY WORDS: Cardiotocography. Fetal heart rate. Fetal well being.

\section{Referências}

1. ACOG practice bulletin. Antepartum fetal surveillance. Number 9, October 1999. Clinical management guidelines for obstetrician-gynecologists. Int $\mathrm{J}$ Gynaecol Obstet 2000; 68:175-85. 
2. Zimmer EZ, Divon MY, Vadasz A. Influence of meperidine on fetal movements and heart rate beat-to-beat variability in the active phase of labor. Am J Perinatol 1988; 5:197-200.

3. Borgatta L, Shrout PE, Divon MY. Reliability and reproducibility of nonstress test readings. Am J Obstet Gynecol 1988; 159:554-8.

4. Gilstrap LC 3rd, Hauth JC, Toussaint S. Second stage fetal heart rate abnormalities and neonatal acidosis. Obstet Gynecol 1984; 63:209-13.

5. Dawes GS, Moulden M, Redman CW. Criteria for the design of fetal heart rate analysis systems. Int J Biomed Comput 1990; 25:287-94.

6. Dawes GS. Computerised analysis of the fetal heart rate. Eur J Obstet Gynecol Reprod Biol 1991; 42 Suppl:S5-8.

7. Dawes GS, Moulden M, Redman CW. Improvements in computerized fetal heart rate analysis antepartum. J Perinat Med 1996; 24:25-36.

8. Street P, Dawes GS, Moulden M, Redman CW. Short term variation in abnormal antenatal fetal heart rate records. Am J Obstet Gynecol 1991; 165:515-23.

9. Dawes GS, Moulden M, Redman CW. Short-term fetal heart rate variation, decelerations, and umbilical flow velocity waveforms before labor. Obstet Gynecol 1992; 80:673-8.

10.Guzman ER, Vintzileos A, Egan JF, Benito C, Lake $\mathrm{M}$, Lai YL. Antenatal prediction of fetal $\mathrm{pH}$ in growth restricted fetuses using computer analysis of the fetal heart rate. J Matern Fetal Med 1998; 7:43-7.

11.Zugaib M, Miyadahira S, Nomura RMY, Francisco RPV. Cardiotocografia computadorizada. In: Zugaib M, Miyadahira S, Nomura RMY, Francisco RPV, editores. Vitalidade Fetal - Propedêutica e Avaliação. $1^{\text {a }}$ ed. São Paulo: Atheneu; 2000. p. 117-25.

12.Flynn AM, Kelly J, Matthews K, O’Connor M, Viegas O. Predictive value of, and observer variability in, several ways of reporting antepartum cardiotocographs. Br J Obstet Gynaecol 1982; 89:434-40.

13. Dawes GS, Redman CW, Smith JH. Improvements in the registration and analysis of fetal heart rate records at the bedside. Br J Obstet Gynaecol 1985; 92:317-25.
14. Ramos JLA. Avaliação do crescimento intra-uterino por medidas antropométricas do recém-nascido [tese]. São Paulo: Universidade de São Paulo. Faculdade de Medicina; 1983.

15.Mariani Neto C. Comparação entre a análise visual e a computadorizada de registros cardiotocográficos anteparto em gestações de alto risco [tese]. Campinas: Universidade Estadual de Campinas. Faculdade de Ciências Médicas; 1999.

16.Smith JH, Dawes GS, Redman CW. Low human fetal heart rate variation in normal pregnancy. $\mathrm{Br} \mathrm{J}$ Obstet Gynaecol 1987; 94:656-64.

17.Economides DL, Selinger M, Ferguson J, Bowell PJ, Dawes GS, Mackenzie IZ. Computerized measurement of heart rate variation in fetal anemia caused by rhesus alloimmunization. Am J Obstet Gynecol 1992; 167:689-93.

18.Dawes GS, Moulden M, Redman CW. Limitations of antenatal fetal heart rate monitors. Am J Obstet Gynecol 1990; 162:170-3.

19.Dawes GS, Lobb MO, Mandruzzato G, Moulden M, Redman CW, Wheeler T. Large fetal heart rate decelerations at term associated with changes in fetal heart rate variation. Am J Obstet Gynecol 1993; 168:105-11.

20.Anceschi MM, Piazze JJ, Vozzi G, et al. Antepartum computerized CTG and neonatal acid-base status at birth. Int J Gynaecol Obstet 1999; 65:267-72.

21.Dawes GS, Meir YJ, Mandruzzato GP. Computerized evaluation of fetal heart-rate patterns. J Perinat Med 1994; 22:491-9.

22.Bracero LA, Morgan S, Byrne DW. Comparison of visual and computerized interpretation of nonstress test results in a randomized controlled trial. Am J Obstet Gynecol 1999; 181:1254-8.

23. Machlitt A, Wauer RR, Chaoui R. Longitudinal observation of deterioration of Doppler parameters, computerized cardiotocogram and clinical course in a fetus with growth restriction. J Perinat Med 2001; 29:71-6.

24.Senat MV, Schwarzler P, Alcais A, Ville Y. Longitudinal changes in the ductus venosus, cerebral transverse sinus and cardiotocogram in fetal growth restriction. Ultrasound Obstet Gynecol 2000; 16:19-24.

Recebido em: 17/4/2001 Aceito com modificações em: 6/12/2001 